\title{
GERMINACIÓN Y EMERGENCIA DIARIA DE CARIÓPSIDES Y DIÁSPORAS DE PASTOS NATIVOS E INTRODUCIDOS
}

\section{DAILY GERMINATION AND EMERGENCE OF CARYOPSES AND DIASPORES IN NATIVE AND INTRODUCED GRASSES}

\author{
Adrián R. Quero-Carrillo', Filogonio J. Hernández-Guzmán ${ }^{2 \star}$, Paulino Pérez- \\ Rodríguez' ${ }^{1}$, Duane Pool ${ }^{3}$, Patricia Landa-Salgado ${ }^{4}$ y Rafael Nieto-Aquino ${ }^{2}$
}

\begin{abstract}
'Campus Montecillo, Colegio de Postgraduados. Km 34.5 Carr. México-Texcoco. 56230, Montecillo, Texcoco, Estado de México. México. Teléfono (595) 9520200 ext. 1712; 1714. ${ }^{2}$ Universidad Politécnica Francisco I. Madero. Domicilio conocido. 42660, Francisco I. Madero, Estado de Hidalgo. México. Tel 01 (738) $7241174 .{ }^{3} \mathrm{CIR}$-Noreste, Campo Experimental San Luis, Instituto Nacional de Investigaciones Forestales, Agrícolas y Pecuarias. Km. 14.5 Carretera San Luis-Matehuala Soledad de Graciano Sánchez. 78430, San Luis Potosí, México. Teléfono: 01 (55) 38718700, ext. 83407. ${ }^{4 D e-}$ partamento de Agroindustrias, Universidad Autónoma Chapingo. Km. 38.5 Carretera México-Texcoco. 56230, Texcoco, Estado de México, México. Tel 01 (595) 9521500
\end{abstract}

*Autor para correspondencia (fjhernandez@upfim.edu.mx)

\section{RESUMEN}

El objetivo del presente trabajo fue evaluar la tasa de germinación diaria (TGD) en cariópsides clasificados por tamaño (CCT) y la tasa de emergencia diaria (TED) tanto en CCT como en diásporas, en los pastos nativos Banderita (Bouteloua curtipendula (Michx.) Torr.) y Navajita (B. gracilis (Willd. ex Kunth) Lag. ex Griffiths), y los introducidos Buffel (Cenchrus ciliaris L.) y Rhodes (Chloris gayana Kunth). Se obtuvieron cariópsides a partir de diásporas y se clasificaron en chicos, medianos y grandes, y todos los propágulos se sometieron a: 1) Pruebas de envejecimiento acelerado (PEA), durante 12, 24 y $36 \mathrm{~h} \mathrm{a} 42^{\circ} \mathrm{C}$ y $100 \%$ de HR. Se sembró a cariópsides sobre papel y a diásporas en Peat Moos a $1 \mathrm{~cm}$ de profundidad y se cuantificó la aparición de plántulas normales durante $15 \mathrm{~d}$ y; 2) Se sembraron los dos propágulos por especie de pasto a $0.5,1.0,2.0,3.0$ y $5.0 \mathrm{~cm}$ de profundidad (PS) en suelos Vertisol y Calcisol y se cuantificó la emergencia durante $64 \mathrm{~d}$. La información se analizó con el procedimiento LIFEREG de SAS $(a=0.05)$. Se observó diferencia en TGD y TED después de PEA para pastos nativos e introducidos $(P \leq 0.001)$. Las TGD mayores en Banderita, Navajita, Buffel y Rhodes fueron a mayor tamaño de cariópside y sin estrés y se concentró a $2 \mathrm{~d}$ después de siembra (dds) mientras en diásporas, en nativos, fueron a menor estrés de 4 a 6 dds y en pastos introducidos a menor estrés, en Buffel, se concentró de 6 a 12 $\mathrm{d}$ y en Rhodes de 5 a $8 \mathrm{~d}$. En cuanto a PS, la mayor TED para las especies estudiadas en cualquier tipo de propágulo, se registró entre 0.5 y $3.0 \mathrm{~cm}$ de PS y, suelo tipo Vertisol mostró mayores TED. Sembrar cariópsides de mayor tamaño reduce el periodo de emergencia de plántulas, lo cual es importante para establecer más plantas por $\mathrm{m}^{2}$ en temporal semiárido.

Palabras clave: Banderita, Navajita, Vertisol, Calcisol, profundidad de siembra.

\section{SUMMARY}

This work evaluated rate of daily germination (RDG) in caryopses classified by size (CCS) and rate of daily emergency (RDE) in both CCS and diaspores in native grasses, Sideoats grama and Blue grama, and introduced grasses, Buffel and Rhodes. Caryopses were obtained from diaspores and classified by size into small, medium and large. All the propagules were tested by 1) accelerated aging tests (AAT) after sowing caryopses on paper and diaspores in peat moss for 12,24 and $36 \mathrm{~h}$ at $42{ }^{\circ} \mathrm{C}$ and $100 \% \mathrm{HR}$, and scoring the number of normal seedlings after $15 \mathrm{~d}$; and 2) successful emergence at different sowing depths, by sowing two propagules of each grass at $0.5,1.0$ 2.0, 3.0 and $5.0 \mathrm{~cm}$ deep in Vertisol and Calcisol soils, and counting the number of plants after $64 \mathrm{~d}$. The data was analyzed through the LIFEREG $(a=$ 0.05 ) SAS procedure. Differences were observed in RDG and RDE after TAA for native and introduced grasses $(P \leq 0.001)$. The highest RDG in Sideoats grama, Blue grama, Buffel grass and Rhodes grass coincided with larger caryopsis, unstressed treatment, and $2 \mathrm{~d}$ after sowing (das); yet in native grasses diaspores, highest RDG was obtained from the minimally-stressed treatment, from 4 to 6 das. For introduced grasses, RDG was greater with the minor stress treatment, and it occurred from 6 to $12 \mathrm{~d}$ in Buffel and from 5 to $8 \mathrm{~d}$ in Rhodes. Sowing depth affected RDE: the highest RDE in any type of propagule was registered between 0.5 and $3.0 \mathrm{~cm}$ planting depth, and Vertisol soil promoted higher RDE. Sowing caryopses of larger sizes reduces emergence length and favors establishment of more plants per $\mathrm{m}^{2}$ under rainfed conditions.

Index words: Sideoats grama, Blue grama, Vertisol, Calcisol, sowing depth.

\section{INTRODUCCIÓN}

La tasa de germinación y emergencia en gramíneas son variables informativas sobre la uniformidad de aparición de plántulas normales (Larsen y Bibby, 2005). En pastos para zonas áridas, la semilla se comercializa como propágulo, diáspora o espiguilla, la cual consiste de cariópside contenido en brácteas accesorias: gluma, lema, palea o ramillas modificadas, dependiendo de la especie. Lo anterior, dificulta el manejo de la siembra, dado que las brácteas accesorias que contienen al cariópside influyen en la pureza física y calidad fisiológica (Enríquez y Quero, 2006). Para tratar de recuperar áreas sobrepastoreadas de pastizal en el Desierto Chihuahuense y Sonorense en México, las semillas deben contar con buena calidad física y fisiológica para expresar su potencial en campo (Cibrián-Tovar et al. 2013), porque durante su almacenamiento, mecanismos de deterioro de ésta disminuyen la germinación y tasa de germinación (Pichardo et al., 2010). 
El inicio de la germinación inicia con la imbibición y con aumentos o modificaciones a hormonas, sobre todo el ácido giberélico, el cual juega un papel importante en alcanzar la terminación de la germinación, al menos en semillas intactas, e incluye también la desactivación del ácido abscísico (Nonogaki et al., 2010). La germinación de un cariópside, de acuerdo con Bewley (1997) y Nonogaki et al., (2010), es la aparición en primer lugar de la radícula y la aparición del mesocótilo o celeoptilo con desarrollo satisfactorio, y la emergencia en pasto Navajita (B. gracilis), según Moreno-Gómez et al. (2012), es la aparición del coleoptilo en la superficie del sustrato, como resultado de su elongación.

La clasificación de cariópsides ha sido estudiada por Larsen y Andreasen (2004) y Springer et al. (2001) en Tripsacum dactyliodes, así como por Ramírez-Calderón et al. (2003) en triticale (Triticum x Secale Wittmack), quienes indican que a mayor peso de cariópside, se presenta mayor tasa de germinación, relación que influye en mayor área foliar y radical, lo cual es de importancia en siembras de temporal (Quian et al., 2006; Tian et al., 2003).

Las pruebas de envejecimiento acelerado (PEA) ayudan a diferenciar calidad entre lotes de semilla, predecir vida de anaquel y se relacionan con emergencia en campo (Wang et al., 2004). Por otro lado, con respecto a profundidad de siembra en pastos, Cox y Martin (1984), Limón y Peco (2016), Quero-Carrillo et al. (2016), Traba et al. (2007), indican que semillas de pasto sembradas de 1 a $2 \mathrm{~cm}$ de profundidad emergerán en mayor porcentaje y disminuirán a mayor profundidad. El objetivo del presente trabajo fue evaluar la velocidad de germinación y emergencia tanto en cariópsides clasificados por tamaño (chico, mediano y grande) como en diásporas de pasto Banderita (Bouteloua curtipendula (Michx.) Torr.), Navajita, Buffel (Cenchrus ciliaris L.) y Rhodes (Chloris gayana Kunth); posterior a tratamientos de 1) envejecimiento acelerado y 2) emergencia a cinco profundidades de siembra en suelos Vertisol y Calcisol.

\section{MATERIALES Y MÉTODOS}

\section{Sitio experimental y material genético}

El experimento se realizó de marzo a julio de 2011 en Montecillo, Estado de México, tanto en laboratorio como en invernadero. El material experimental consistió de propágulos (semilla) de dos especies de pasto nativas del Desierto Chihuahuense: Navajita y Banderita variedad Reno, así como dos especies introducidas de África: Rhodes, variedad Bell y Buffel, variedad T4464. La semilla utilizada fue comercial, la cual se caracterizó inicialmente para contenido de humedad y prueba de viabilidad con tetrazolio (0.1
$\%$; ISTA, 2017); lo anterior, con el fin de uniformizar la tasa de germinación y emergencia por especie y tamaño de cariópside. Los experimentos tanto en laboratorio como en invernadero se realizaron en tres ocasiones, en laboratorio con intervalo de $30 \mathrm{~d}$ y en invernadero de $64 \mathrm{~d}$. En cada ocasión que se realizó el experimento, se obtuvieron 200 g de cariópsides de manera manual mediante fricción con tapete y almohadilla de caucho corrugado a partir de diásporas comerciales. Para clasificar cariópsides por tamaño (CCT) se midió el grosor de 200 semillas botánicas y después se separó con ayuda de tamices, y se conservaron en sobres de papel en condiciones de laboratorio $\left(20 \pm 3^{\circ} \mathrm{C}\right)$.

\section{Fase de laboratorio}

Para envejecimiento acelerado (PEA), los propágulos (cariópsides y diásporas) permanecieron en estufa $\left(42^{\circ} \mathrm{C}\right.$, $100 \%$ HR) por 12, 24 y 36 h y una vez concluido el tiempo de estrés (TE), se lavó el material biológico y el testigo (sin estrés) con hipoclorito de sodio (0.6\%) por 3 min e inmediatamente se sumergió a la semilla en Captan ( $1 \mathrm{~g} \mathrm{~L}^{-1}$ agua) por 3 min y se enjuagó con agua destilada. Posteriormente, se sembró por especie, CCT o diásporas y el testigo (CCT y diásporas, sin estrés), en cuatro repeticiones de 100 unidades de dispersión con base en semilla pura viable (SPV; ISTA, 2017). Los sustratos incluyeron: a) Papel filtro, para sembrar por CCT en caja plástica transparente $(20 \times 15 \times 8$ $\mathrm{cm}$ ) con flujo ligero de aire; b) Peat Moss, para sembrar diásporas a $1 \mathrm{~cm}$ de profundidad en charolas de 40×30×5 $\mathrm{cm} \sin$ tapa. Las siembras se colocaron en cámara de ambiente controlado a $22{ }^{\circ} \mathrm{C}$ con luz fluorescente durante las $24 \mathrm{~h}$. Para regar a las siembras por CCT, se aplicaron $30 \mathrm{~mL}$ de agua destilada en el papel filtro a cada una, cada $48 \mathrm{~h}, \mathrm{y}$ para diásporas $100 \mathrm{~mL}$ por aspersión sobre el Peat Moss, cada 72 h. La germinación de plántulas normales para CCT y diásporas se consideró efectiva cuando fue evidente la primera hoja, y se contabilizó cada 24 h; en CCT, hasta 15 d después de siembra (dds), y en diásporas hasta 25 dds (ISTA, 2017).

\section{Fase de invernadero}

Los CCT, diásporas y charolas (con 70 orificios individuales; $4 \times 2 \times 10 \mathrm{~cm}$ ), se desinfectaron por inmersión en solución de hipoclorito de sodio al $0.6 \%$ durante 3 min; posteriormente, se enjuagaron con agua destilada y se secaron a la sombra. Los sustratos utilizados fueron suelos tipo Calcisol de Salinas Hidalgo, San Luis Potosí (INEGI, 2014a) y Vertisol de Atotonilco El Grande, Hidalgo (INEGI, 2014b). Los suelos se sometieron a esterilización en autoclave durante $6 \mathrm{~h}$ para evitar germinación de semilla de maleza. La siembra en ambos suelos se realizó a capacidad de campo a profundidades de $0.5,1.0,2.0,3.0$ y $5.0 \mathrm{~cm}$ en charolas de unicel 
y cada cavidad (unidad experimental) midió $10 \times 3.5 \mathrm{~cm}$, después se sembró con SPV, para obtener cinco plántulas por unidad experimental (seis cariópsides para tamaño mediano y grande en las cuatro especies; siete cariópsides en tamaño chico en Banderita y ocho en Navajita, Buffel y Rhodes). Se regó a saturación cada 48 h con agua potable. La cuantificación de plántulas se realizó cada 24 h por 64 d, para CCT y diásporas, y fue efectiva cuando la primera hoja fue evidente.

\section{Tratamientos evaluados y variables respuesta}

En laboratorio, para la PEA los tratamientos incluyeron la combinación de tres CCT en dos especies nativas o introducidas por cada tiempo de estrés (TE) (0, 12, 24 o 36 h), i.e., 24 tratamientos para las especies nativas y 24 para las introducidas; por otra parte, en diásporas, la combinación de dos pastos nativos o introducidos en cada TE, lo cual resultó en ocho tratamientos para cada grupo de especies. Las variables respuesta fueron la tasa de germinación diaria (TGD) y la tasa de emergencia diaria (TED)

En invernadero los tratamientos resultaron de la combinación de cinco diferentes profundidades de siembra (PS), dos especies nativas o introducidas, tres tipos de CCT sembradas en dos tipos suelo, i.e., 60 tratamientos por grupo; mientras que, para diásporas, los tratamientos resultaron de la combinación de dos especies nativas o dos introducidas sembradas en cinco PS y en dos tipos de suelo, lo que resultó en 20 tratamientos para especies nativas y 20 para introducidas. La variable respuesta evaluada fue la tasa de emergencia diaria tanto para cariópsides como para diásporas.

\section{Análisis estadístico}

Los datos se analizaron por regresión logística (Hosmer y Lemeshow, 2000) mediante procedimientos para análisis de tiempos de vida (LIFEREG) de SAS (SAS Institute, 2009). La comparación de la germinación en el tiempo se realizó por razón de verosimilitudes generalizada $(a=0.05)$, con base en los resultados de la rutina LIFEREG. Con la finalidad de comparar las curvas de germinación en el tiempo se utilizaron modelos estadísticos comúnmente utilizados en análisis de tiempos de vida, donde se presentan datos censurados. El modelo utilizado es: $\log \left(T_{i}\right)=x_{i}^{\prime} \beta+\sigma e_{i}$, donde $T_{i}$ es el tiempo a germinación, $x_{i}^{\prime}$, representa un vector de covariables asociadas al tiempo de germinación (por ejemplo, tamaños de semilla, tratamiento, etc), $\beta$ representa un vector de parámetros de regresión asociados a las covariables, $\sigma$ es un parámetro de escala positivo y $e_{i}$ es un error aleatorio.

\section{RESULTADOS Y DISCUSIÓN}

La humedad determinada en las unidades de dispersión completas (diásporas: glumas, lemas, paleas, aristas y ramillas, más cariópside) de los pastos utilizados, estuvo en el rango adecuado en condiciones de almacenamiento, lo cual ayuda a reducir su deterioro y pérdida de vigor (Pichardo et al., 2010; Cuadro 1). En cuanto al grosor de cariópsides, Banderita y Rhodes tienen mayor proporción de cariópsides medianos, Buffel y Navajita de cariópsides grandes (Cuadro 1).

\section{Germinación y emergencia posterior al envejecimiento acelerado}

Se observaron diferencias en TGD tanto en CCT como en diásporas de pastos nativos e introducidos, a nivel inter e intra especie $(P \leq 0.001)$. Una mayor TGD se observó para nativos a 2 dds y a menor TE $(P \leq 0.001)$; para Banderita (Cuadro 2), en CCT chico, mediano y grande se ob-

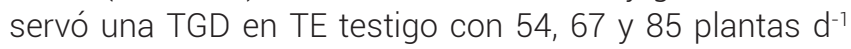
$(P<0.001)$; mientras que en Navajita se registró 39, 43 y 69 plantas $\mathrm{d}^{-1}$ con $\mathrm{P} \leq 0.001$, respectivamente, lo que indica que a mayor CCT, se registró mayor TGD y por lo tanto, mayor vigor (Ramírez-Calderón et al., 2013). Por otro lado, en diásporas sembradas en Peat Moss en laboratorio, la mayor TED posterior a PEA mostró diferencias $(P \leq 0.001)$, en Banderita y Navajita ocurrió a 5 dds y fue de mayor a menor TE. Por lo anterior, en pastos nativos las diásporas empiezan su emergencia hasta 5 dds, mientras que los cariópsides concentran la germinación a 2 dds, lo que puede ser de utilidad en siembras en campo porque las raíces disponen de $3 \mathrm{~d}$ adicionales para explorar el suelo en busca de humedad (Tian et al., 2002).

En pastos introducidos (Cuadro 3), la mayor TGD en Buffel $(P \leq 0.001)$ se observó en cariópsides grandes, a 2 dds en el testigo (40 plantas $\left.\mathrm{d}^{-1}\right)$, mientras que en cariópsides chicos y medianos, a 3 dds y 12 h de TE ( 26 plantas $\mathrm{d}^{-1}$ ) y en el testigo (32 plantas $d^{-1}$ ), respectivamente. En Rhodes, la mayor TGD fue a 2 dds para cariópsides grandes, medianos y chicos a $12 \mathrm{~h}$ de TE $(\mathrm{P} \leq 0.001 ; 81,80$ y 71 plantas $\mathrm{d}^{-1}$, respectivamente). Con respecto a la TED en diásporas, sembradas en Peat Moss en laboratorio, se observó diferencia $(P \leq 0.001)$, Buffel inició a 5 dds, pero el periodo de emergencia se amplió hasta 12 dds; cabe mencionar que la TED mayor ocurrió en el testigo (13 plantas $\mathrm{d}^{-1}$ ). En pasto Rhodes, la TED mayor ocurrió 5 dds en el testigo (38 plantas $\mathrm{d}^{-1}$ ) y se observó un periodo de emergencia, de 4 a 9 dds. Por tanto, eliminar brácteas florales incrementó la aparición de las estructuras visibles de la germinación con respecto a la diáspora completa, como fue reportado por Tian et al. (2002). Lo anterior, es una oportunidad para rápido establecimiento dada la alta 
Cuadro 1. Humedad de unidades de dispersión completas de semillas de cuatro especies de pastos, número de cariópsides en 100 diásporas y grosor de cariópsides para su clasificación.

\begin{tabular}{lccccccc}
\hline \multirow{2}{*}{$\begin{array}{l}\text { Nombre común } \\
\text { de pasto }\end{array}$} & $\begin{array}{c}\text { Humedad } \\
(\%)\end{array}$ & \multicolumn{3}{c}{ Tamaños de tamiz $(\mathrm{mm})$} & \multicolumn{3}{c}{ Proporción (\%) } \\
\cline { 3 - 7 } & 11.4 & $0.5-0.58$ & $0.59-0.69$ & $\geq 0.79$ & 11 & 78 & 11 \\
\hline Banderita & 12.3 & $0.5-0.58$ & $0.59-0.69$ & $\geq 0.79$ & 11 & 33 & 56 \\
Buffel & 11.4 & $0.42-0.49$ & $0.50-0.58$ & $\geq 0.59$ & 11 & 36 & 53 \\
Navajita & 11.4 & $0.42-0.49$ & $0.50-0.58$ & $\geq 0.59$ & 31 & 50 & 19 \\
Rhodes & & & & & & & Chicos \\
\hline
\end{tabular}

velocidad de germinación y desarrollo del área subcoleoptilar, la cual contiene el meristemo generador de raíces adventicias que sustentan la supervivencia de plantas adultas (Moreno-Gómez et al., 2012); sin embargo, lotes de bajo vigor, implican bajo porcentaje de plantas en campo (Wang et al., 2004), como es el caso del lote de pasto Navajita.

\section{Tasa de emergencia diaria por tamaño de cariópside y diásporas en pastos nativos en invernadero}

Se observó diferencia en la TED para cariópsides clasificados por tamaño (CCT) y diásporas a nivel inter e intra especie ( $\mathrm{P} \leq 0.001$; Figura 1). La mayor TED ocurrió a mayor tamaño de cariópside en Banderita y Navajita, lo cual es importante para mejorar la selección de genotipos que contengan mayor proporción de cariópsides grandes al establecer praderas de temporal en agostaderos degradados (Moreno-Gómez et al., 2012; Quero-Carrillo et al., 2016).

En promedio de las cinco profundidades de siembra (PS) en CCT de Banderita de 8 a 12 dds (periodo de mayor emergencia de plántulas) se observó diferencia entre tamaños de cariópside ( $P \leq 0.001$; Figura 1a). Las mayores TED ocurrieron de 6 a 12 dds, para cariópsides chicos, medianos y grandes (2, 3 y 4 plantas $\mathrm{d}^{-1}$ ), respectivamente; mientras que en Navajita (Figura 1b) las mayores TED fueron de 7 a 11 dds $\left(1,1\right.$ y 1 planta $\left.\mathrm{d}^{-1}\right)$, respectivamente.

Se observó mayor TED en diásporas de Banderita con 6 plantas $\mathrm{d}^{-1}$ a 9 y $11 \mathrm{dds}$ y, en Navajita 5 plantas $\mathrm{d}^{-1}$ a $7 \mathrm{dds}$ $(P \leq 0.001)$; la mayor TED de Navajita se registró en diásporas y no en cariópsides ( 5 vs. 1 planta $\mathrm{d}^{-1}$ ); por lo tanto, no es conveniente eliminar las brácteas en pasto Navajita.

\section{Tasa de emergencia diaria por tamaño de cariópside y diásporas en pastos introducidos}

Se observó diferencia en TED a profundidad de siembra de $0.5,1.0,2.0,3.0$ y $5.0 \mathrm{~cm}$ por tamaño de propágulo en pastos introducidos a nivel inter e intra especie $(P \leq$ 0.001; Figura 2) y el periodo de emergencia fue de 1 a 14 dds, mientras que Larsen y Bibby (2004) en T. dactyloides reportaron de 5 a 24 dds; sin embargo, eliminar brácteas accesorias en pasto Buffel mejora la TED en $2 \mathrm{~d}$ y en Rhodes en $4 \mathrm{~d}$, lo que en campo puede favorecer la carrera contra la pérdida de humedad en el suelo durante la sequía intraestival y competencia con maleza de hoja ancha y pastos anuales (Cibrián-Tovar et al., 2013).

El mayor periodo de TED en pruebas de PS para los propágulos de pasto Buffel y Rhodes en cariópside chico, mediano, grande y diásporas fue de 6 a 14 dds; con 2, 2, 3 y 2 plantas $\mathrm{d}^{-1}$; y 4, 1, 2 y 2 plantas $\mathrm{d}^{-1}$, respectivamente $(\mathrm{P} \leq$ 0.001 ; Figura 2). La mayor TED en pasto Buffel se observó en diásporas a 11 dds con 6 plantas $\mathrm{d}^{-1}$ y en CCT en cariópside chico a 9 dds con 5 plantas $\mathrm{d}^{-1}$; en pasto Rhodes, la mayor TED se registró en cariópside grande a 7 dds con 7 plantas $\mathrm{d}^{-1}$. El pasto Rhodes registró mayor TED en comparación con pasto Buffel $(P \leq 0.001)$, quizá por la persistencia de inhibidores (ácido abscísico y cafeínico) en este último.

\section{Tasa de emergencia diaria a diferente profundidad de siembra de propágulo}

La TED a diferentes PS para pasto Banderita en CCT presentó los valores mayores a $0.5,1.0$ y $2.0 \mathrm{~cm}$ en el periodo

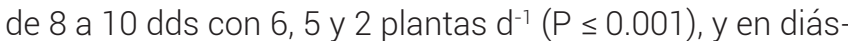
poras de 11 a 13 dds con 5, 6 y 3 plantas $d^{-1}(P \leq 0.001)$, respectivamente. Por otro lado, en pasto Navajita se registraron las mayores TED en cariópsides de 0.5 a $1.0 \mathrm{~cm}$ de PS en el periodo de 8 a 10 dds (6 y 5 plantas $d^{-1} ; P \leq 0.001$ ), mientras que en diásporas de pasto Navajita, las mayores TED ocurrieron a 0.5, 1.0, 2.0 y $3.0 \mathrm{~cm}$ en el periodo de $11 \mathrm{a}$ 13 dds $\left(7,6,4\right.$ y 4 plantas $\left.d^{-1} ; P \leq 0.001\right)$; lo que indica que en pasto Navajita no conviene eliminar brácteas accesorias en siembras de temporal. Con respecto a lo anterior, Quian et al. (2006) registraron un periodo de germinación hasta 73 dds en laboratorio con Distichlis spicata, y Larsen y Bibby (2004) registraron la aparición de plántulas de 1 a 25 dds con cariópsides de T. dactyloides. Por lo tanto, el pasto Navajita es más susceptible a mayor PS en comparación con Banderita, por lo que estos valores bajos de TED 


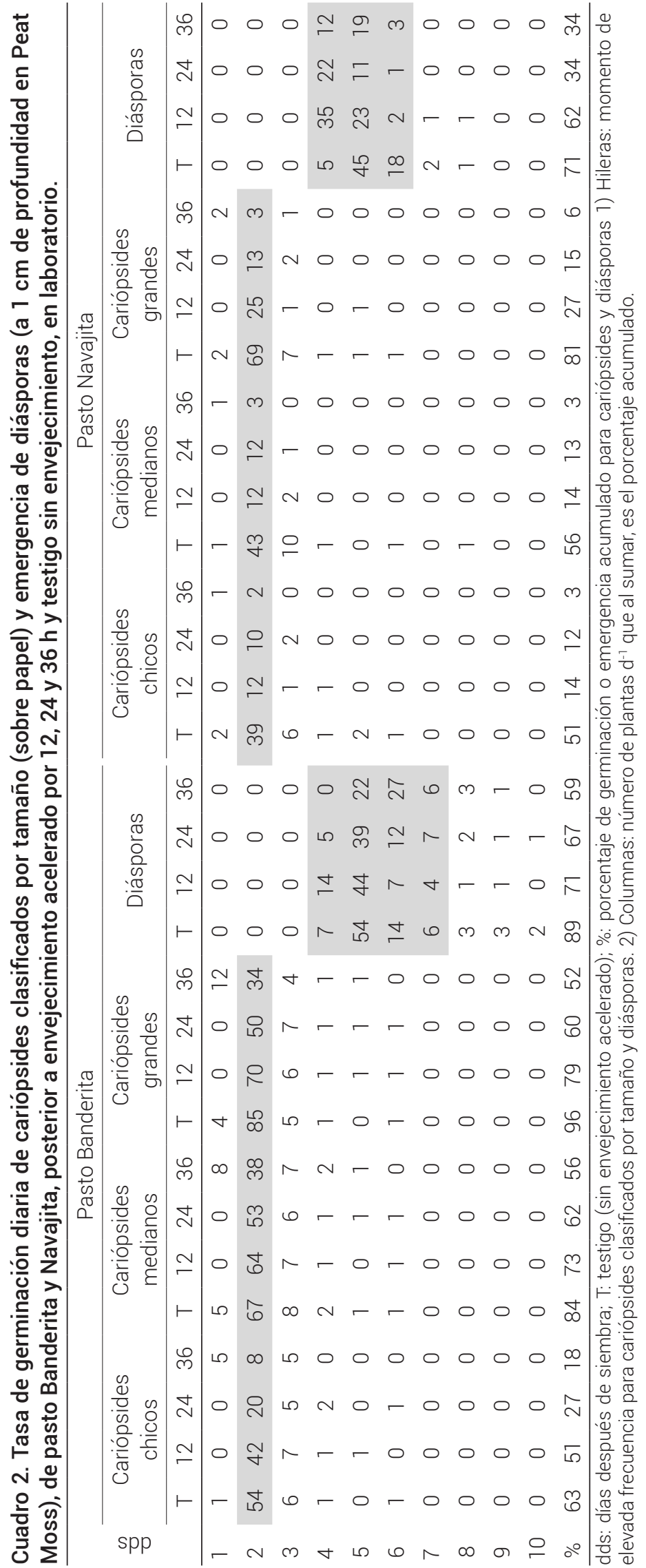

pueden ser por vigor bajo, debido a procesos de cosecha y almacenamiento inadecuados, lo que repercute en emergencia heterogénea de plántulas normales (Martínez et al., 2010). Las mayores TED ocurrieron en ambos pastos nativos a 0.5, 1.0 y $2.0 \mathrm{~cm}$, en concordancia con lo reportado por Cox y Martin (1984).

Las TED en pastos introducidos fueron diferentes para cada tipo de propágulo y entre propágulo a cada PS $(\mathrm{P} \leq$ 0.001). En pasto Buffel se observó el periodo de emergencia de 5 a 19 dds para CCT $(P \leq 0.001)$ y en diásporas de 7 a 27 dds ( $P \leq 0.001$; Figura 3a.3); por su parte el pasto Rhodes en CCT fue de 5 a 14 dds y en diásporas de 7 a 20 dds ( $P \leq 0.001$; Figura 3.b.3), lo que puede ser explicado por la presencia de latencia (Quero-Carrillo et al., 2016) que no permite expresar la emergencia en un periodo más corto de tiempo. Las mayores TED en pasto Buffel y Rhodes fueron variables $(P \leq 0.001)$; sin embargo, para CCT fue de 0.5 a $2.0 \mathrm{~cm}$ y para diásporas de 0.5 a $3.0 \mathrm{~cm}$ de PS. En pasto Buffel en CCT la mayor TED ocurrió a $1.0 \mathrm{~cm}$ de 14 a 16 dds $\left(6\right.$ plantas $\mathrm{d}^{-1}$ ) y en diásporas a 1.0 y $2.0 \mathrm{~cm}$ de 11 a $13 \mathrm{dds}$ (6 y 6 plantas $\left.\mathrm{d}^{-1}\right)$, respectivamente.

Por lo anterior Limón y Peco (2016) señalan que durante los primeros días de lluvias el tiempo es muy valioso, porque posterior a la emergencia sobrevive menos del $35 \%$ de plántulas; por su parte, Tian et al. (2002), en T. dactyloides, indican que al remover brácteas y escarificar cariópsides la germinación aumenta, lo que en el presente estudio fue muy notable en los pastos Banderita, Buffel y Rhodes.

\section{Tasa de emergencia por tipo de suelo en pastos nativos e introducidos}

Se observó diferencia en TED tanto en pastos nativos como introducidos en dos tipos de suelo $(P \leq 0.001$; Figura 4). En pasto Banderita (Figura 4.a.1), el periodo de emergencia en suelo Vertisol fue de 6 a 12 dds y en Calcisol de 9 a 20 dds ( $P \leq 0.001)$; sin embargo, en siembras con diásporas a 8 dds, la mayor TED ocurrió en suelo Vertisol (Figura 4.b. 1; 10 plantas $\mathrm{d}^{-1}$ ). En pasto Navajita la TED (Figura 4.a. 1) fue afectada negativamente en suelo Calcisol tanto para cariópsides como diásporas (Figura 4.b.1; P $\leq 0.001$ ), porque suelos de diferente textura ocasionan variaciones en la emergencia. Rivera et al. (2012) mencionan que la tasa de aparición de plántulas es afectada por el tipo, profundidad y humedad del suelo, y Moreno-Gómez et al. (2012) mencionan que los suelos arcillosos con pobre materia orgánica dificultan el desarrollo del coleoptilo y después el de la primera hoja, lo cual, coincide con este estudio.

En pastos introducidos se observó diferencia en TED a nivel inter e intra propágulo $(P \leq 0.001)$. El periodo de emergencia en CCT en suelo Vertisol y Calcisol en pasto Buffel 


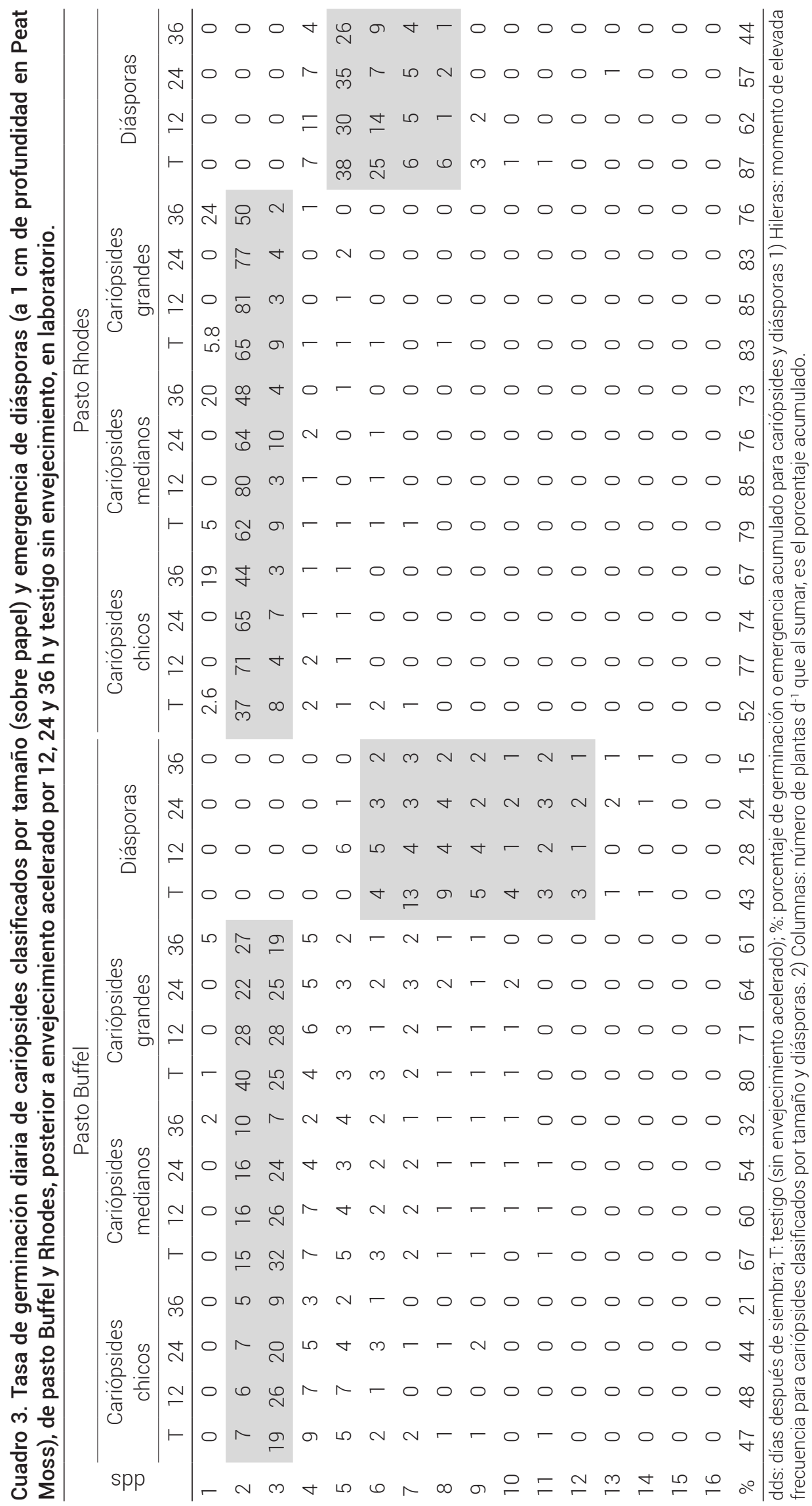



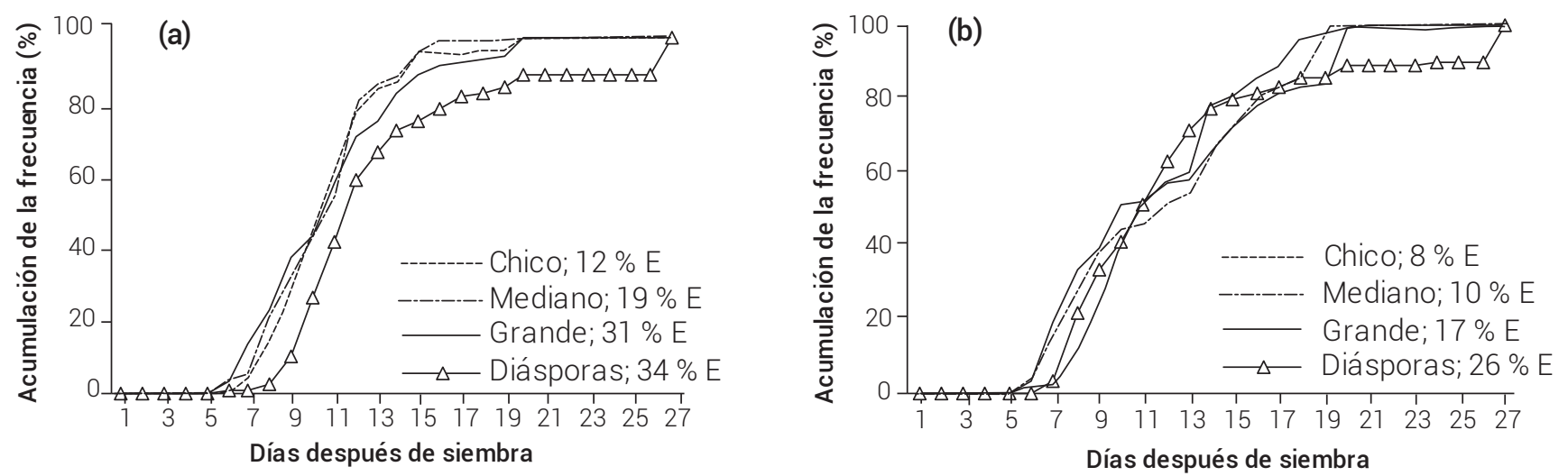

Figura 1. Tasa de emergencia diaria promedio de tres tamaños de cariópside y diásporas de plántulas de pasto Banderita (a) y Navajita (b). Experimento realizado en condiciones de invernadero. E: porcentaje de emergencia acumulada durante el experimento.
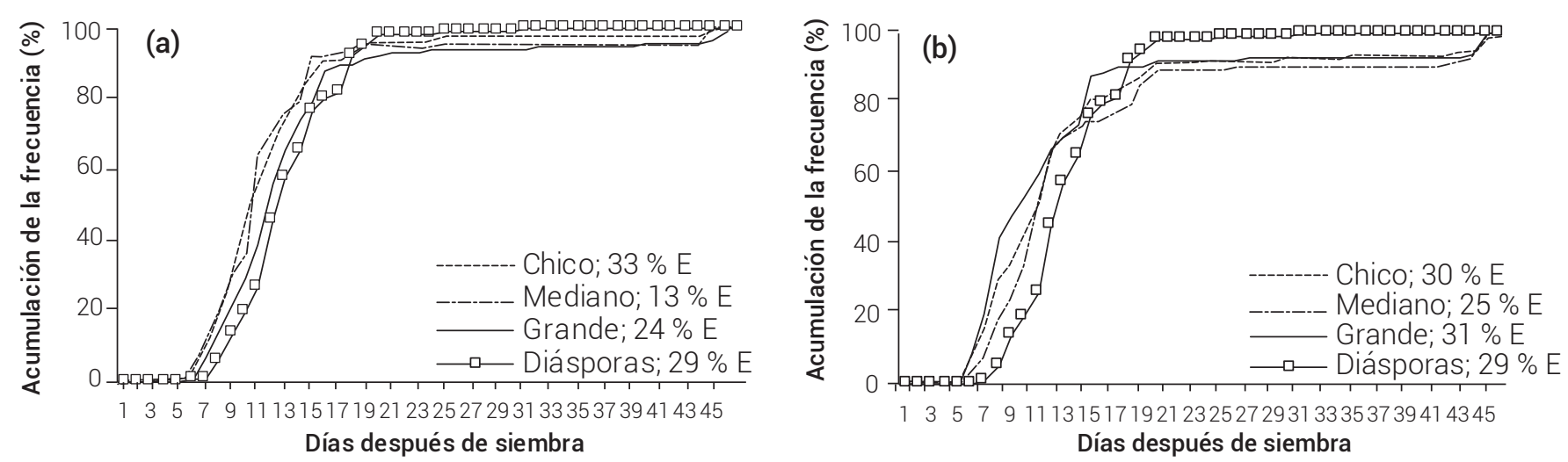

Figura 2. Tasa de emergencia diaria promedio de tres tamaños de cariópside y diásporas de plántulas de dos pastos introducidos de África a México, Buffel (a) y Rhodes (b). Experimento realizado en condiciones de invernadero. E: porcentaje de emergencia acumulada durante el experimento.

fue de 6 a 15 dds y de 6 a 19 dds (Figura 4.a.2; $P \leq 0.001$ ), respectivamente, y por su parte, en diásporas de 7 a 20 dds y de 9 a 20 dds (Figura 4.b.2), respectivamente; por su parte TED en Rhodes en cariópsides fue de 6 a 20 dds y de 6 a 19 dds (Figura 4.a.2) y en diásporas de 7 a 26 dds y de 9 a 24 dds (Figura 4.b.2), respectivamente, por lo tanto en ambas especies de pasto la TED es afectada en suelo Calcisol pero en menor medida en comparación con pastos nativos como fue consignado por Cox y Martin (1984), donde pastos de origen africano se establecieron en suelos de Arizona en mejor manera en comparación con pastos nativos, mientras que Rivera et al. (2012) y Traba et al. (2007) mencionan que el tipo de suelo y la textura de éste influyen en la emergencia de plántulas y su posterior establecimiento, porque dependerá de la materia orgánica y preparación de terreno para que las raíces realicen exploración en busca de humedad y puedan sobrevivir a la sequía intraestival.

\section{CONCLUSIONES}

Las mejores germinaciones de propágulos sometidos a envejecimiento acelerado en pasto Banderita y Navajita ocurrieron a mayor tamaño de cariópside y a menor tiempo de estrés por envejecimiento acelerado y se concentró a 2 dds, mientras que la emergencia en sustrato Peat Moss fue de 4 a 6 dds. En pastos introducidos, en Buffel las germinaciones mayores ocurrieron a mayor tamaño de cariópside y sin estrés por envejecimiento acelerado de 2 a 5 dds y con diásporas sin estrés de 6 a 10 dds; en Rhodes las germinaciones mayores con cariópsides fueron de 2 a 3 dds a mayor tamaño de cariópside y sin estrés por envejecimiento acelerado, y con diásporas de 4 a 6 dds y sin estrés.

Las mayores tasas de emergencia diaria en pastos nativos, a diferentes profundidades de siembra, ocurrieron en 

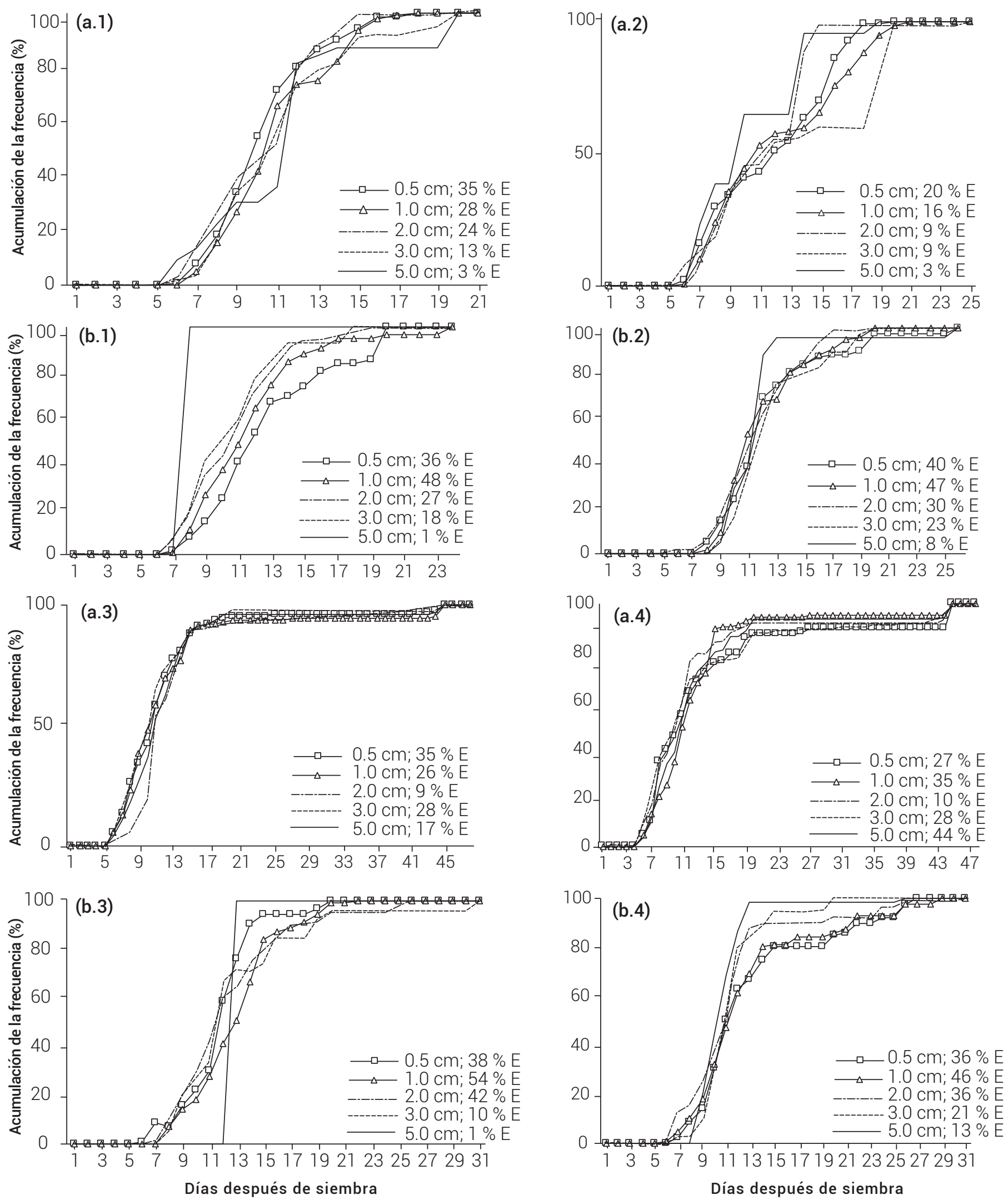

Figura 3. Tasa de emergencia diaria promedio a $0.5,1.0,2.0,3.0$ y $5.0 \mathrm{~cm}$ de profundidad de siembra de cariópsides clasificados por tamaño (a) y diásporas (b) en invernadero para pasto Banderita (1), Navajita (2), Buffel (3) y Rhodes (4). E: porcentaje de emergencia acumulada durante el experimento. 

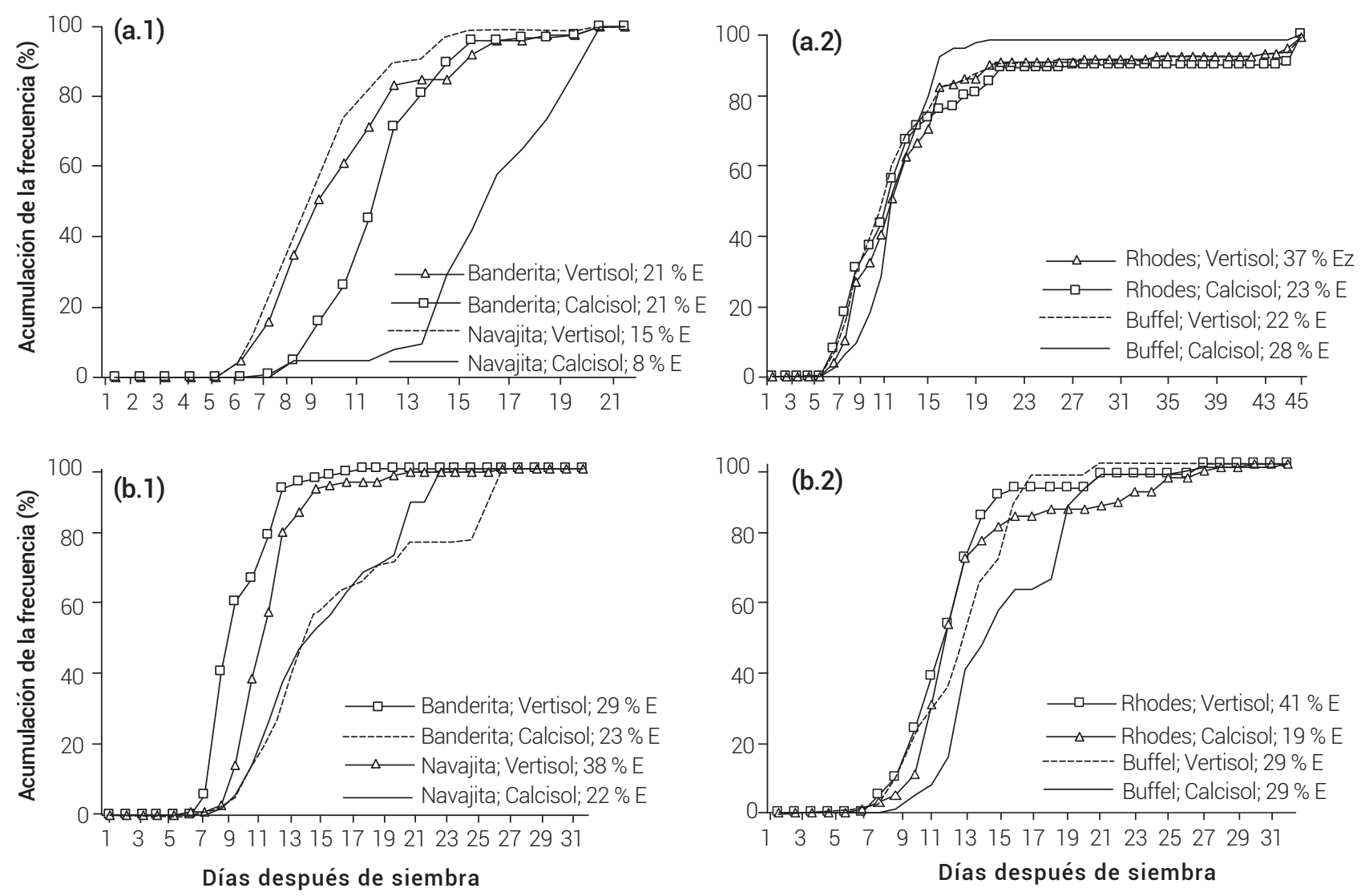

Figura 4. Tasa de emergencia diaria promedio en cariópsides (a) y diásporas (b) en pastos nativos (1) e introducidos (2) y suelo Vertisol o Calcisol, en invernadero. E: emergencia acumulada (\%).

pasto Banderita de 0.5 a $3.0 \mathrm{~cm}$ y en Navajita de 0.5 a 2.0 $\mathrm{cm}$ y la emergencia inició a los 7 dds. El pasto Banderita superó en $5 \mathrm{~d}$ a la mostrada por diásporas, mientras que en Navajita no es conveniente eliminar estructuras florales. En suelo Vertisol se observaron mayores emergencias diarias en comparación a Calcisol. En relación con pastos introducidos, en Buffel la mayor tasa de emergencia diaria ocurrió con cariópsides de 0.5, 2.0 y 3.0 cm; por su parte en pasto Rhodes fue de igual manera con cariópsides de 1.0 a $2.0 \mathrm{~cm}$. La eliminación de brácteas accesorias en los pastos Banderita, Buffel y Rhodes es una alternativa para promover la germinación con rápido desarrollo.

\section{BIBLIOGRAFÍA}

Bewley J. D. (1997) Seed germination and dormancy. The Plant Cell 9:1055-1066

Cibrián-Tovar J., A. Quero-Carrillo, M. Muñiz-Merino, F. J. Hernández-Guzmán, A. Cibrián-Jaramillo y M. Manzano-Camarillo (2013) Nanotecnología agrícola: Caso 1. Liberadores de feromonas, Caso 2. Establecimiento de gramíneas en condiciones semiáridas. Agroproductividad 6:3-8

Cox J. R. and M. H. Martin (1984) Effects of planting depth and soil texture on the emergence of four lovegrasses. Journal of Range Managment 37:204-205.

Enríquez Q. J. F. y Quero C. A. R. (2006) Producción de Semillas de Gramí- neas y Leguminosas Forrajeras Tropicales. Libro Técnico Núm. 11. Campo Experimental Cotaxtla. Instituto Nacional de Investigaciones Forestales, Agrícolas y Pecuarias. Veracruz, México. $109 \mathrm{p}$.

Hosmer D. W. and S. Lemeshow (2001) Applied Logistic Regression. 2nd ed. Wiley-Interscience, New York. 375 p.

INEGI, Instituto Nacional de Geografía, Estadística e Informática (2014a) Anuario estadístico y geográfico de San Luis Postosí. San Luis Potosí, México. 611 p

INEGI, Instituto Nacional de Geografía, Estadística e Informática (2014b) Anuario estadístico y geográfico de Hidalgo. Pachuca de Soto, Hidalgo. 611 p.

ISTA, International Seed Testing Association (2017) International Rules for Seed Testing. International Seed Testing Association. Zurich, Switzerland. 296 p.

Larsen S. U. and C. Andreasen (2004) Light and heavy turfgrass seeds differ in germination percentage and mean germination thermal time. Crop Science 44:1710-1720.

Larsen S. U. and B. M. Bibby (2004) Use of germination curves to describe variation in germination characteristics in three turfgrass species. Crop Science 44:891-899.

Larsen S. U. and B. M. Bibby (2005) Differences in thermal time requirement for germination of three turfgrass species. Crop Science 45:2030-2037.

Limón A. and B. Peco (2016) Germination and emergence of annual species and burial depth: Implications for restoration ecology. Acta Oecologica 71:8-13.

Martínez S. J., J. Virgen V., M. G. Peña 0. y A. Santiago R. (2010) Índice de velocidad de emergencia en líneas de maíz. Revista Mexicana de Ciencias Agrícolas 1:289-304

Moreno-Gómez B., E. García-Moya, Q. Rascón-Cruz y G. A. Aguado-Santacruz 
(2012) Crecimiento y establecimiento de plántulas de Bouteloua gracilis (Kunth) Lag. ex Griffiths y Eragrostis curvula var. conferta Stapf bajo un régimen simulado de lluvia. Revista Fitotecnia Mexicana 35:299-308.

Nonogaki H., G. W. Bassel and J. D. Bewley (2010) Germination still a mystery. Plant Science 179:574-581.

Pichardo G. J. M., O. J. Ayala G., V. A. González H., C. M. Flores 0., J. A. Carrillo S., A. Peña L., A. Robledo P. y G. García S. (2010) Calidad fisiológica, ácidos grasos y respiración en semillas de tomate de cáscara deterioradas artificialmente. Revista Fitotecnia Mexicana 33:231-238.

Quero-Carrillo A. R., F. J. Hernández-Guzmán, M. Velázquez-Martínez, H. G. Gámez-Vázquez, P. Landa-Salgado y P. Aguilar-López (2016) Métodos de establecimiento de pasturas en zonas áridas de México utilizando semillas crudas o cariópsides. Tropical GrasslandsForrajes Tropicales 4:29-37.

Quian Y. L., J. A. Cosenza, S. J. Wilhelm and D. Christensen (2006) Techniques for enhancing saltgrass seed germination and establishment. Crop Science 46:2613-2616.

Ramírez-Calderón J. J., T. Cervantes-Santana, H. E. Villaseñor-Mir y C. LópezCastañeda (2003) Selección para componentes del rendimiento de grano en triticale irradiado. Agrociencia 37:595-603.

Rivera D., B. M. Jáuregui and B. Peco (2012) The fate of herbaceous seeds during topsoil stockpiling: restoration potential of seed banks Ecological Engineering 44:94-101.

SAS Institute (2009) SAS/STAT User quide version 9.2. SAS Institute Inc. Cary, North Carolina, USA. 5136 p.

Springer T. L., C. L. Dewald and G. E. Aiken (2001) Seed germination and dormancy in Eastern gamagrass. Crop Science 41:1906-1910.

Tian X., A. D. Knapp, K. J. Moore, E. C. Brummer and T. B. Bailey (2002) Cupule removal and caryopsis scarification improves germination of Eastern gamagrass seed. Crop Science 42:185-189.

Tian X., A. D. Knapp, L. R. Gibson, R. Struthers, K. J. Moore, E. C. Brummer and T. B. Bailey (2003) Response of Eastern gamagrass seed to gibberellic acid buffered below its pKa. Crop Science 43:927-933.

Traba J., F. M. Azcárate and B. Peco (2007) From what depth do seeds emerge? A soil seed bank experiment with Mediterranean grassland species. Seed Science Research 14:297-303.

Wang Y. R., L. Yu, Z. B. Nan and Y. L. Liu (2004) Vigor tests used to rank seed lot quality and predict field emergence in four forage species. Crop Science 44:535-541. 